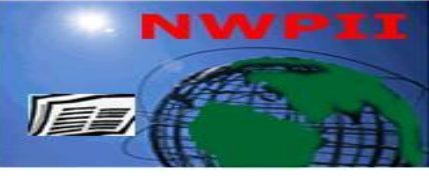

ISSN: 1937-9080 nwpii.com/ajbms

\title{
Chemosenstizing Effects of a Novel Anti-inflammatory Small Molecule, UTL-5g, and Its Analogs
}

\author{
B. Chen ${ }^{1}$, W-H. Huang ${ }^{2}$, A-R. Lee ${ }^{2}$, J. Media ${ }^{3}$, F. Valeriote, J. Shaw ${ }^{1, *}$ \\ ${ }^{1} 21^{\text {st }}$ Century Therapeutics, Inc., Detroit, Michigan 48202 \\ ${ }^{2}$ National Defense Medical Center, Taipei, Taiwan \\ ${ }^{3}$ Henry Ford Health Systems, Detroit, Michigan 48202 \\ *Corresponding author \\ Jiajiu Shaw, Ph.D. \\ $21^{\text {st }}$ Century Therapeutic, Inc. \\ 440 Burroughs St., Suite 447 \\ Detroit, Michigan 48202 \\ USA \\ E-mail: jiajiushaw@gmail.com \\ Phone (734) 330-6052
}

Received: 2 July 2015; | Revised: 17 September 2015; | Accepted: 24 September 2015

\section{Abstract}

Our previous studies showed that UTL-5g, a novel anti-inflammatory TNF- $\alpha$ inhibitor, enhanced the chemotherapeutic efficacy of cisplatin against HCT-15 human colon cancer cells in an animal tumor model. To extend the study, we set out to investigate 3 newly synthesized analogs of UTL-5g (UTLOH-4a, -4b, and $-4 \mathrm{c})$ on whether they are associated with similar biological effects. Chemosensitizing effects of UTL-5g and its 3 analogs were investigated using HCT-15 cells in vitro with increasing doses of cisplatin, carboplatin, or oxaliplatin. $\mathrm{IC}_{50}$ of each drug was determined from the dose-response surviving curves. The antiinflammatory effects were examined by their ability to inhibit $\mathrm{PGE}_{2}$ production in mouse RAW cells stimulated with LPS. Myelotoxicity induced by cisplatin was examined with human CFU-GM clonogenic assay. All 4 compounds significantly potentiated the cytotoxic effect of cisplatin against HCT-15 cancer cells by lowering the $\mathrm{IC}_{50}$ values. Among them, UTL-5g appeared to be the most promising sensitizer of cisplatin and UTLOH-4b was the most potent sensitizer of carboplatin. Anti-inflammatory study showed that UTL-5g, UTLOH-4a, and -4c inhibited PGE 2 production in LPS-stimulated RAW cells. In addition, UTL-5g and UTLOH-4a enhanced the survival of CFU-GM colony counts in cultures containing cisplatin, carboplatin and oxaliplatin respectively.

Keywords: chemosensitizer, cisplatin, HCT-15, CFU-GM, UTL-5g, PGE 2 


\section{Introduction}

One of the several ways to improve chemotherapy is to use chemosensitizers to potentiate anti-cancer drugs against cancer cells. Two potential beneficial outcomes of using ideal chemosensitizers are: 1) the same dose of an anticancer drug may be used to have a higher cancer killing effect without increasing its side effects, and 2) a lower dose of an anticancer drug may be used to achieve the same chemotherapeutic effect but with fewer/lower side effects. For a widely prescribed anti-cancer drug, cisplatin, a number of potential chemosensitizers have been reported in various tumor models and leukemia.

Chemosensitizers may be divided into two classes: synthetic compounds and naturally occurring compounds [1-10] and they may act with the same or different mechanisms of action. Despite the significant progress over the years, most chemosensitizers are known to be associated with undesirable side effects such as myelotoxicity and/or other additional toxicities. Therefore, research in the area of chemosensitization continues to be an area of interest.

Tissue microenvironment with chronic inflammation has been shown to promote malignant transformation and cancer development $[11,12]$. In general, the longer the inflammation persists, the higher the risk of cancer. For example, inflammatory bowel disease with constant inflammation is well established as a risk factor for colorectal carcinoma development. Inflammatory mediators such as metabolites of arachidonic acid, inflammatory cytokines, and free radicals may be responsible for cancer promoting effects. For example, $\mathrm{PGE}_{2}$ has been shown to be overproduced in many types of tumors and is known to promote tumor progression $[13,14]$. A number of earlier studies suggest that anti-inflammatory agents may act as potential chemsensitizers. For example, quinacrine, an antirheumatic agent, was reported to enhance cisplatin-induced cytotoxicity in four cancer cell lines [15]. Saikawa et al. showed that pretreatment of TR-5 colon cancer cells with a COX-2 inhibitor, JTE-522, restored the chemosensitivity of TR-5 cells to cisplatin [3]. Furthermore, Yu et al. showed that inhibition of $\mathrm{NF \kappa B}$ pathway by berberine enhances chemosensitivity to irinotecan in HCT-116 human colon cancer cells [16]. Clinical studies from randomized trials in patients and in animal models showed that long term treatment with anti-inflammatory agents is not only chemopreventive but also increases the sensitivity of chemotherapy against colorectal cancer [17].
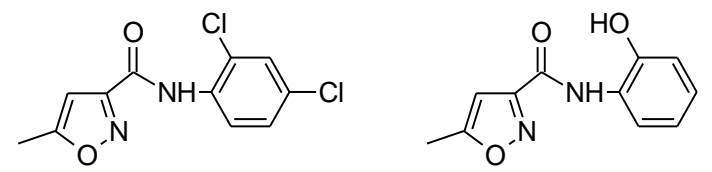

UTL-5g

UTLOH-4a

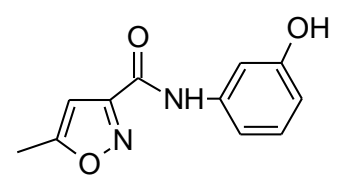

UTLOH-4b

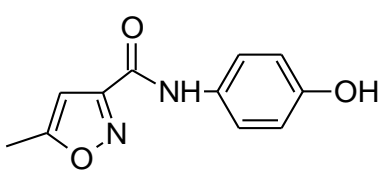

UTLOH-4c

Figure 1: Structures of four selected potential chemosensitizers

In our previous animal studies, we showed that UTL-5g, a tumor necrosis factor-alpha (TNF$\alpha$ ) inhibitor, exhibited potent anti-inflammatory and antirheumatic activities [18]. Subsequently, we showed that UTL-5g potentiated chemotherapeutic efficacy of cisplatin against 
human colon cancer cells HCT-15 in an animal tumor model. In addition, UTL-5g acted as a potential chemoprotector by reducing side effects induced by cisplatin [19]. In this study, we investigated the chemosensitizing effect of 3 newly synthesized UTL-5g analogs (Figure 1). We now report that these UTL-5g analogs express similar chemosensitizing effect against HCT-15 colon cancer cells in vitro. In addition, we show that two of these analogs and UTL-5g are potent inhibitor of $\mathrm{PGE}_{2}$ production. Likewise, these new compounds reduce cisplatininduced myelotoxicity in an in vitro model using human bone marrow CFU-GM assay.

\section{Materials and Methods}

\subsection{Reagents}

UTL-5g (HPLC purity >98\%) was provided by Dr. Jiajiu Shaw. UTLOH-4a, $-4 b$, and $-4 c$ (HPLC purity $>98 \%$ ) were provided by Dr. WenHsin Huang at School of Pharmacy, National Defense Medical Center, Taipei, Taiwan. Cisplatin, carboplatin and oxaliplatin were purchased from Alfa Aesar (Ward Hill, MA) without further purification. Recombinant human granulocyte-macrophage colony-stimulating factor (rhGM-CSF) was purchased from Stemcell Technologies (Vancouver, Canada). Recombinant human macrophage colony-stimulating factor (rhM-CSF) was obtained from PeproTech (Rocky Hill, NJ). $\beta$-mercaptoethanol (BME) and other chemicals were purchased from Sigma Chemical (St. Louis, MO).

\subsection{Cells}

Human colon cancer cells, HCT-15, were obtained from ATCC (Manassas, VA) and maintained under standard cell culture conditions in RPMI 1640 medium (MediaTech Inc, Manassas, VA) containing $10 \%$ bovine calf serum (BCS) (HyClone, Logan, UT), 1\% Lglutamine $(200 \mathrm{mM}), 100 \mu \mathrm{g} / \mathrm{mL}$ streptomycin and 100 units/mL penicillin (MediaTech Inc. Manassas, VA). Human bone marrow cells were purchased from Lonza Walkersville (Walkersville MD). Upon arrival, human bone marrow cells were washed once with Hank's Balanced Salt Solution (HBSS) and resuspended in alpha-MEM containing $20 \% \mathrm{BCS}, 1 \% \mathrm{~L}$ glutamine $(200 \mathrm{mM}), 100 \mu \mathrm{g} / \mathrm{mL}$ streptomycin and 100 units $/ \mathrm{mL}$ penicillin. Cell counts were obtained by using a working solution of $0.4 \%$ trypan blue in PBS and a hemocytometer.

\subsection{IC $_{50}$ Determination}

Briefly, $10^{5}$ HCT-15 cancer cells were seeded in a T-25 flask containing $5 \mathrm{~mL}$ of the culture medium and incubated in a $5 \% \mathrm{CO}_{2}$ incubator at $37{ }^{\circ} \mathrm{C}$ for three days. Each flask was added with a testing compound (final concentration $2 \mu \mathrm{M}$ ) for $30 \mathrm{~min} 37{ }^{\circ} \mathrm{C}$. Thereafter, cell cultures were treated with increasing doses of cisplatin, carboplatin or oxaliplatin (from 0 to $20 \mu \mathrm{g} / \mathrm{mL}$ in saline) respectively and incubated for an additional 5 days. Each sample was trypsinized, centrifuged to obtain a pellet, decanted, resuspended in HBSS, and then stained with trypan blue for dead and viable cell count. $\mathrm{IC}_{50}$ was determined from the dose-response surviving curve from each culture.

\subsection{Prostaglandin E2 (PGE $)$ assay}

The anti-inflammatory effect was investigated by their ability to inhibit the production of $\mathrm{PGE}_{2}$ in LPS-stimulated mouse RAW cells. RAW (264.7) mouse macrophage/monocytic cell line was obtained from ATCC (Manassas, VA). They were routinely grown in Dulbecco's modified eagle medium (DMEM) supplemented with $10 \%$ heat inactivated BCS [20, 21]. Briefly, RAW cells $\left(2 \times 10^{5} / \mathrm{mL}\right.$ in 24 -well culture plate) were treated with increasing doses of each testing compound for $1 \mathrm{hr}$ at $37{ }^{\circ} \mathrm{C}$. Thereafter, cells were challenged with $20 \mu \mathrm{L} /$ well of LPS (equivalent to a final concentration of 100 ng/mL). Control included RAW cell cultures containing the testing compounds and culture without LPS stimulation in each experiment. Cells were then further cultured at $37{ }^{\circ} \mathrm{C}$ overnight. Culture supernatants were removed from each well and centrifuged at 14,000 g for 15 min. Levels of $\mathrm{PGE}_{2}$ in the supernatants were determined using commercial ELISA assay kit obtained from R\&D Systems (Minneapolis, MN) in triplicate. Optical density of each well was determined using a BioTek Epoch microplate reader (Winooski, VT) set to $450 \mathrm{~nm}$. 


\subsection{Human CFU-GM Clonogenic Assay}

Myelotoxicity induced by cisplatin was examined with human CFU-GM clonogenic assay. Briefly, $8.8 \mathrm{~mL}$ culture medium containing $20 \%$ BCS, $10 \mathrm{ng} / \mathrm{ml} \mathrm{rhGM-CSF,} 10 \mathrm{ng} / \mathrm{ml} \mathrm{rhM}-$ CSF, $5 \times 10^{-5} \mathrm{M}$ mercaptoethanol and 300,000 human bone marrow cells in a $15 \mathrm{~mL}$ culture tube was mixed with $100 \mu \mathrm{L}$ of a test compound or vehicle (final $0.2 \%$ DMSO) and then incubated in a $5 \% \mathrm{CO}_{2}$ incubator at $37^{\circ} \mathrm{C}$ for $30 \mathrm{~min}$. After pretreatment, each tube was mixed with $100 \mu \mathrm{L}$ of cisplatin $(100 \mu \mathrm{g} / \mathrm{mL}$ in saline $)$ or $100 \mu \mathrm{L}$ saline (medium control) individually. Immediately thereafter, each tube was added with $1 \mathrm{~mL}$ of $3 \%$ agar, mixed and transferred into three $60 \mathrm{~mm}$ culture dish $(3 \mathrm{~mL} / \mathrm{dish}$ in triplicate). The dishes were incubated at $37^{\circ} \mathrm{C}$ for seven days and colonies were counted using a dissecting microscope (Bausch \& Lomb, Rochester, NY) at $40 \mathrm{x}$. Cell clusters containing 50 or more cells were considered as colonies.

\section{Results and Discussion}

\subsection{Chemosensitization in vitro}

The $\mathrm{IC}_{50}$ values of 3 platinum drugs on HCT15 human colon cancer cells, with and without pretreatment of a test compound, were determined by using the protocol described in Materials and Methods. It was found that pretreatment of UTL-5g (at $2 \mu \mathrm{M}$ ) on HCT-15 cells significantly lowered the $\mathrm{IC}_{50}$ values of two platinum drugs (cisplatin and oxaliplatin) and the potentiation effect on cisplatin is particularly impressive ( $\mathrm{IC}_{50}$ reduced to one fourth) (Table 1). UTL-5g by itself has a low anticancer activity against HCT-15 with $\mathrm{IC}_{50}$ of $>34 \mu \mathrm{M}$ in vitro. The results are consistent with and complement our previous studies in which we showed that UTL-5g potentiated the chemotherapeutic efficacy of cisplatin in animal studies [19].

The chemosensitization effects of UTLOH$4 \mathrm{a},-4 \mathrm{~b}$, and $-4 \mathrm{c}$ were carried out with similar approach. As shown in Table 2, pretreatment of HCT-15 cells with UTL-5g analogs (at $2 \mu \mathrm{M}$ ) for 30 min markedly reduced the $\mathrm{IC}_{50}$ values of cisplatin against HCT-15 cells (3 - 4 fold reduction of that without pretreatment), similar to that treated with UTL-5g. However, for carboplatin, only two of these four compounds (UTLOH-4b and -4c) showed significant chemosensitization against HCT-15 cells, and the reduction of $\mathrm{IC}_{50}$ values of carboplatin were more than that of cisplatin. The most impressive observation is that UTLOH- $4 \mathrm{~b}$ was the most potent sensitizer against HCT-15 cells with the $\mathrm{IC}_{50}$ reduced from $3.5 \mu \mathrm{g} / \mathrm{mL}$ to $0.23 \mu \mathrm{g} / \mathrm{mL}$, a 15 fold reduction. However, UTL-5g (Table 1) and UTLOH-4a (Table 2) did not significantly potentiate carboplatin against HCT-15 cells.

Table 1: $\mathrm{IC}_{50}$ values of cisplatin, carboplatin, and oxaliplatin against HCT-15 cells in vitro with and without pretreatment of UTL-5g $(2 \mu \mathrm{M})$

\begin{tabular}{ccc}
\hline \multirow{2}{*}{ Cytotoxic agents } & \multicolumn{2}{c}{$\mathrm{IC}_{50}(\mu \mathrm{g} / \mathrm{mL})$} \\
\cline { 2 - 3 } & No Pretreatment & UTL-5g $(2 \mu \mathrm{M})^{*}$ \\
\hline Cisplatin & 1.7 & 0.42 \\
Carboplatin & 3.5 & 3.2 \\
Oxaliplatin & 6.8 & 3.2 \\
\hline
\end{tabular}

*HCT-15 cells were pretreated with UTL-5g for 30 min prior to the addition of cytotoxic agents. $\mathrm{IC}_{50}$ was determined from the dose-response curves of each culture.

\section{2 $\mathrm{PGE}_{2}$ study}

We have shown that UTL-5g is a potent antiinflammatory agent in previous studies. The antiinflammatory effect of UTL-5g analogs was investigated by their ability to suppress $\mathrm{PGE}_{2}$ production in mouse RAW cells stimulated with LPS (Fig. 2). UTLOH-4a and UTLOH-4c showed inhibitory effect on $\mathrm{PGE}_{2}$ production by 
RAW cells. The inhibitory activity was doesdependent; at $25 \mu \mathrm{M}$ these compounds suppressed approximately $80 \%$ of $\mathrm{PGE}_{2}$ production by RAW cells. UTL-5g also showed similar inhibitory activity. In contrast, UTLOH$4 \mathrm{~b}$ did not inhibit $\mathrm{PGE}_{2}$ production by RAW cells even at the highest dose $(50 \mu \mathrm{M})$ used in the study (Fig. 2). No appreciable amount of $\mathrm{PGE}_{2}$ was detected in cultures without LPS stimulation. The effects of these compounds in lowering elevated $\mathrm{PGE}_{2}$ levels are in line with the antiinflammatory property of UTL-5g reported previously.

Table 2: $\mathrm{IC}_{50}$ values of cisplatin and carboplatin against HCT-15 cells in vitro with and without the pretreatment of UTL-5g and its 3 analogs respectively

\begin{tabular}{ccccc}
\hline \multirow{2}{*}{ Cytotoxic agents } & \multicolumn{4}{c}{$\mathrm{IC}_{50}(\mu \mathrm{g} / \mathrm{mL})^{*}$} \\
\cline { 2 - 5 } & None & UTLOH-4a & UTLOH-4b & UTLOH-4c \\
\hline Cisplatin & 1.7 & 0.57 & 0.59 & 0.60 \\
Carboplatin & 3.5 & 3.1 & 0.23 & 0.55 \\
\hline
\end{tabular}

*HCT-15 cells were pretreated with the analogs $(2 \mu \mathrm{M})$ for 30 min followed with increasing doses of cisplatin and carboplatin (See: Materials and methods).

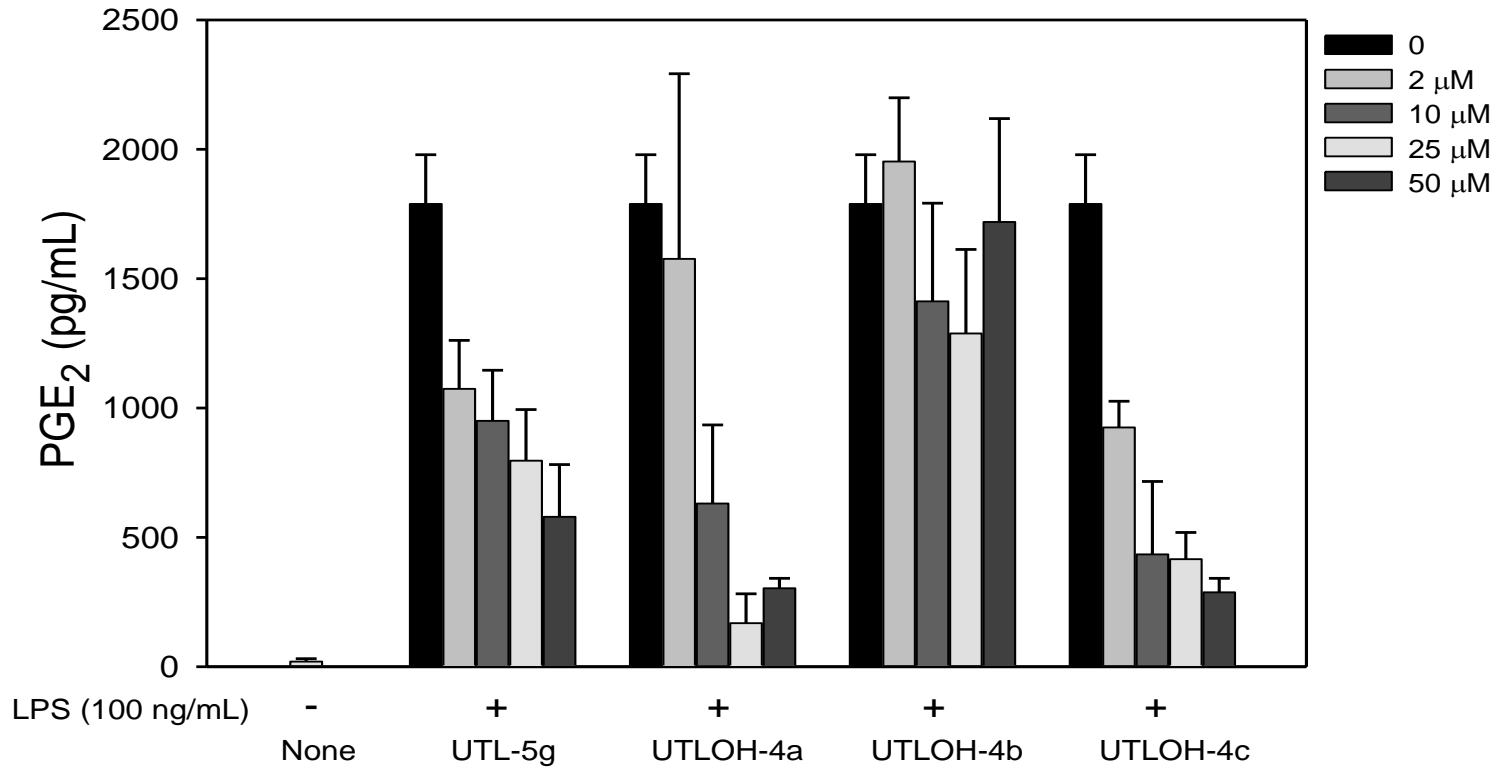

Figure 2: Inhibition of $\mathrm{PGE}_{2}$ production by UTL-5g and its analogs.

Mouse RAW cells $\left(2 \times 10^{5} / \mathrm{mL} /\right.$ well $)$ were treated with UTL-5g, UTLOH-4a, $-4 \mathrm{~b}$, and $-4 \mathrm{c}$ respectively as indicated for $60 \mathrm{~min}$ followed by stimulation with LPS $(100 \mathrm{ng} / \mathrm{mL})$ for an additional $16 \mathrm{hr}$. Levels of $\mathrm{PGE}_{2}$ in supernatants were measured using $\mathrm{PGE}_{2}$ ELISA kit. Data represent mean \pm S.D. from two experiments. 


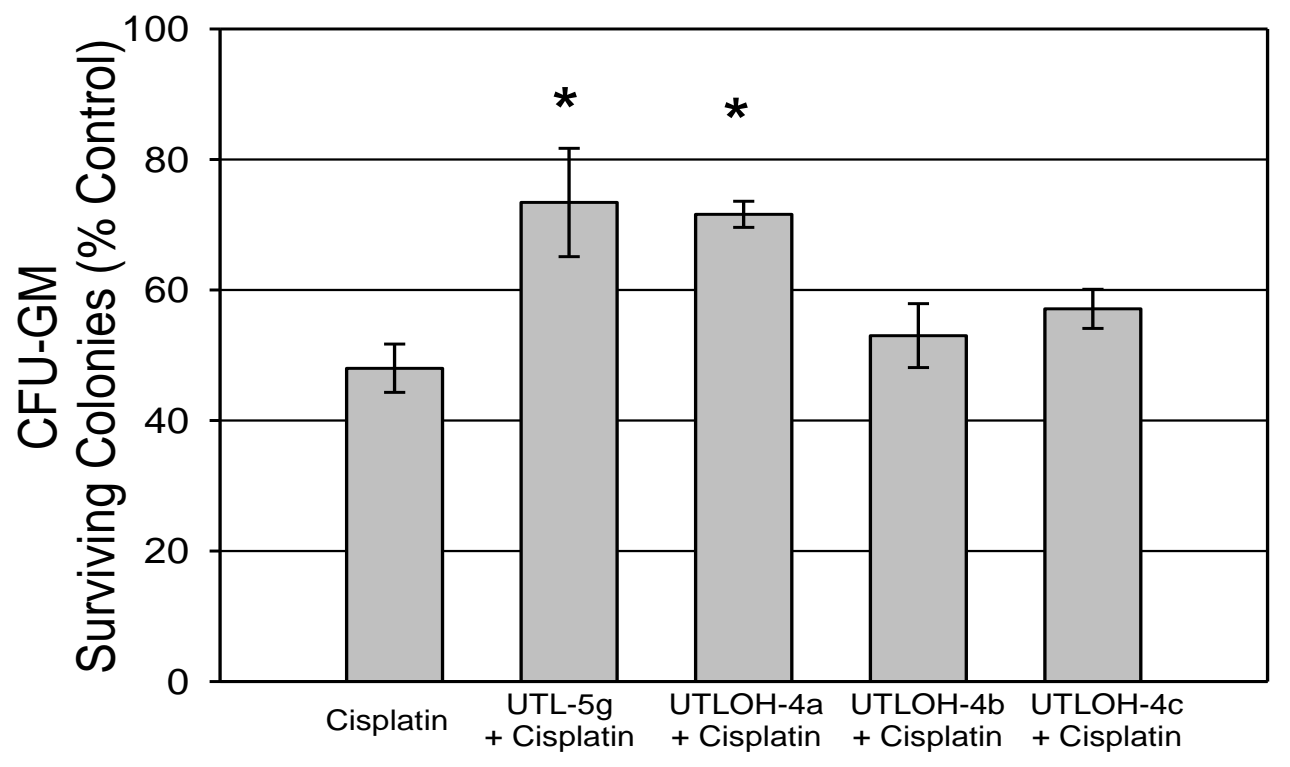

Figure 3: Reduction of cisplatin-induced myelotoxicity by UTL-5g and its analogs.

Cisplatin final concentration was $1 \mu \mathrm{g} / \mathrm{mL}$. The final concentration of UTL-5g, UTLOH-4a, $-4 \mathrm{~b}$, or-4c final concentration was $1 \mu \mathrm{M}$. ${ }^{*} \mathrm{p}=0.05$ (two-tailed, paired) for UTL-5g pretreatment vs. cisplatin only and UTLOH-4a pretreatment vs. cisplatin only. Data are mean \pm S.D. from three experiments.

It is now well established that chronic inflammation is associated with malignant transformation and cancer development $[11,12]$. One of the major inflammatory mediators, $\mathrm{PGE}_{2}$, has been shown to be overproduced in many types of tumors and is known to promote tumor progression [13, 14]. Results from epidemiological studies, animal model studies, and randomized trials in patients showed that long term treatment with anti-inflammatory agent is potential chemopreventive against colorectal cancer and increases the sensitivity of chemotherapy [17]. Thus, the chemosensitizing effect of UTL-5g and its analogs may be linked to their anti-PGE 2 activity shown in this study. However, one of the analog, UTLOH-4b, showed potent chemosensitizing effect but did not inhibit $\mathrm{PGE}_{2}$ production. Thus, detailed studies in animal tumor models is needed to further understand the mechanisms of these anti-inflammatory compounds in promoting chemotherapeutic efficacy to anti-cancer drugs.

\subsection{Reduction of Myelotoxicity in vitro - Chemoprotection}

The effect of UTL-5g and its analogs on cisplatin-induced myelotoxicity was examined using human bone marrow CFU-GM clonogenic assay. Results from clonogenic assay showed that none of these compounds enhanced the cytotoxic effect of cisplatin against bone marrow cells. On the other hand, two of them, UTL-5g and UTLOH-4a, actually reduced the toxicity on bone marrow cells induced by cisplatin. As shown in Fig. 3, cisplatin $(1 \mu \mathrm{g} / \mathrm{mL})$ reduced the bone marrow CFU-GM colony count to $49 \%$ (relative to medium control). Yet, pretreatment of bone marrow cells with UTL-5g or UTLOH-4a prior to cisplatin treatment increased the surviving colony counts to $74 \%$ and $73 \%$, respectively, indicating that both UTL-5g and UTLOH-4a could protect CFU-GM against cisplatin induced cytotoxicity. The pretreatment of UTLOH-4b and $-4 c$ also increased the surviving colonies of CFU-GM although not statistically significant. For these compounds, the order of bone marrow cell 
protection against cisplatin was UTL-5g UTLOH-4a > UTLOH-4c > UTLOH-4b.

\section{Summary}

In summary, our results showed that UTL-5g analogs showed several biologic activities similar to their parental compound. Like UTL-5g, these compounds could act as chemosensitizers, promoting the anti-cancer effects of cisplatin, carboplatin, and/or oxaliplatin. In addition, these compounds did not enhance the myelotoxicity of cisplatin against normal human bone marrow progenitor cells and may protect them from the toxicity of cisplatin as shown in CFU-GM assay. In addition, two of the analogs show potent inhibitory activity against $\mathrm{PGE}_{2}$ production. Thus, while the exact mechanisms are not clear at present, the anti-inflammatory property of UTL$5 \mathrm{~g}$ and its analogs may contribute at least in part to both chemosensitization and chemoprotection effects observed in this study [19].

\section{Acknowledgements}

This work was supported in part by NIH/NCI grant 5R44CA141749-03.

\section{Abbreviations}

CFU-GM, colony forming unit for both granulocyte and macrophage; COX-2, Cyclooxygenase-2; $\quad \mathrm{PGE}_{2}, \quad$ LPS, lipopolysaccharide; prostaglandin E2; rhM-CSF, recombinant human macrophage colony stimulating factor; rhGM-CSF, recombinant human granulocyte macrophage colony stimulating factor

\section{References}

1. Tsutsui, K.; Komuro, C.; Ono, K.; Nishidai, T.; Shibamoto, Y.; Takahashi, M.; Abe, M. Chemosensitization by buthionine sulfoximine in vivo, Int J Radiat Oncol Biol Phys, 1986, 12,1183-1186. PMID 2427490
2. Chen, G.; Zeller, W.J. Augmentation of cisplatin (DDP) cytotoxicity in vivo by DLbuthionine sulfoximine (BSO) in DDPsensitive and-resistant rat ovarian tumors and its relation to DNA interstrand cross links, Anticancer Res, 1991, 11,2231-2237. PMID 1776864

3. Saikawa, Y.; Sugiura, T.; Toriumi, F.; Kubota, T.; Suganuma, K.; Isshiki, S.; Otani, Y.; Kumai, K.; Kitajima, M. Cyclooxygenase-2 gene induction causes CDDP resistance in colon cancer cell line, HCT-15, Anticancer Res, 2004, 24,27232728. PMID 155178787

4. Walling, J.M.; Stratford, I.J. Chemosensitization by monofunctional alkylating agents, Int J Radiat Oncol Biol Phys, 1986, 12,1397-1400. PMID 2759563

5. Keane, T.E.; Rosner, G.L.; Gingrich, J.R.; Poulton, S.H.; Walther, P.J. The therapeutic impact of dipyridamole: chemopotentiation of the cytotoxic combination 5fluorouracil/cisplatin in an animal model of human bladder cancer, J Urol, 1991, 146,1418-1424. PMID 1942313

6. Adams, C.; McCarthy, H.O.; Coulter, J.A.; Worthington, J.; Murphy, C.; Robson, T.; Hirst, D.G. Nitric oxide synthase gene therapy enhances the toxicity of cisplatin in cancer cells, J Gene Med, 2009, 11,160-168. DOI: $10.1002 /$ jgm. 1280

7. Li, Q.Q.; Wang, G.; Reed, E.; Huang, L.; Cuff, C.F. Evaluation of cisplatin in combination with beta-elemene as a regimen for prostate cancer chemotherapy, Basic Clin Pharmacol Toxicol, 2010, 107,868-876. DOI: 10.1111/j.1742-7843.2010.00592.x

8. Gupta, S.C.; Kannappan, R.; Reuter, S.; Kim, J.H.; Aggarwal, B.B. Chemosensitization of tumors by resveratrol, Ann N Y Acad Sci, 2011, 1215,150-160. DOI: 10.1111/j.1749-6632.2010.05852.x

9. Rajesh, D.; Stenzel, R.A.; Howard, S.P. Perillyl alcohol as a radio-/chemosensitizer in malignant glioma, J Biol Chem, 2003, 278,35968-35978.

DOI: 10.1074/jbc.M303280200

10. Goel, A.; Aggarwal, B.B. Curcumin, the golden spice from Indian saffron, is a 
chemosensitizer and radiosensitizer for tumors and chemoprotector and radioprotector for normal organs, Nutr Cancer, 2010, 62,919-930. DOI: 10.1080/01635581.2010.509835

11. Landskron, G.; De la Fuente, M.; Thuwajit, P.; Thuwajit, C.; Hermoso, M.A. Chronic inflammation and cytokines in the tumor microenvironment, J Immunol Res, 2014, 2014,149185. DOI: $10.1155 / 2014 / 149185$

12. Shacter, E.; Weitzman, S.A. Chronic inflammation and cancer, Oncology (Williston Park), 2002, 16,217-226, 229; discussion 230-212. PMID 11866137

13. Schrey, M.P.; Patel, K.V. Prostaglandin E2 production and metabolism in human breast cancer cells and breast fibroblasts. Regulation by inflammatory mediators, $\mathrm{Br} \mathrm{J}$ Cancer, 1995, 72,1412-1419. PMID 8519653

14. Sinha, P.; Clements, V.K.; Fulton, A.M.; Ostrand-Rosenberg, S. Prostaglandin E2 promotes tumor progression by inducing myeloid-derived suppressor cells, Cancer Res, 2007, 67,4507-4513. DOI: 10.1158/0008-5472.CAN-06-4174

15. Wang, Y.; Bi, Q.; Dong, L.; Li, X.; Ge, X.; Zhang, X.; Fu, J.; Wu, D.; Li, S. Quinacrine enhances cisplatin-induced cytotoxicity in four cancer cell lines, Chemotherapy, 2010, 56,127-134. DOI: 10.1159/000313525

16. Yu, M.; Tong, X.; Qi, B.; Qu, H.; Dong, S.; Yu, B.; Zhang, N.; Tang, N.; Wang, L.; Zhang, C. Berberine enhances chemosensitivity to irinotecan in colon cancer via inhibition of NFkappaB, Mol Med Rep, 2014, 9,249-254. DOI: 10.3892/mmr.2013.1762

17. Chu, A.J.; Chou, T.H.; Chen, B.D. Prevention of colorectal cancer using COX-2 inhibitors: basic science and clinical applications, Front Biosci, 2004, 9,26972713. PMID 15353307

18. Shaw, J.; Chen, B.; Wooley, P.; Huang, W.H.; Lee, A.R.; Zeng, D. Antiinflammatory and Anti-arthritic Effects of a Novel Leflunomide Analogue, UTL-5b (GBL-5b), Am J Biomed Sci, 2011, 3,31-39. DOI: 10.5099/aj110100031

19. Shaw, J.; Chen, B.; Huang, W.H.; Lee, A.R.; Media, J.; Valeriote, F.A. The smallmolecule TNF-alpha modulator, UTL-5g, reduces side effects induced by cisplatin and enhances the therapeutic effect of cisplatin in vivo, J Exp Ther Oncol, 2011, 9,129-137. PMID 21699020

20. Ralph, P.; Nakoinz, I. Antibody-dependent killing of erythrocyte and tumor targets by macrophage-related cell lines: enhancement by PPD and LPS, J Immunol, 1977, 119,950954. PMID 894031

21. Raschke, W.C.; Baird, S.; Ralph, P.; Nakoinz, I. Functional macrophage cell lines transformed by Abelson leukemia virus, Cell, 1978, 15,261-267. PMID 212198 